\title{
Overexpression of miR-34a and miR-16 Synergistically Promotes Apoptosis in Breast Cancer Cells
}

\author{
Mehdi Haghi ${ }^{1,2,{ }^{*}}$, Arash Javeri ${ }^{1}$, Masoumeh Fakhr Taha ${ }^{1}$ \\ ${ }^{1}$ Department of Stem Cells and Regenerative Medicine, Institute for Medical \\ Biotechnology, National Institute of Genetic Engineering and Biotechnology \\ (NIGEB), Tehran, Iran \\ ${ }^{2}$ Islamic Azad University, Damghan Brach, Damghan, Iran \\ * Corresponding Author: Mehdi Haghi, Islamic Azad University, Damghan Brach, \\ Damghan, Iran, Email: mehdihaghi11@gmail.com
} DOI: $10.21859 / \mathrm{mci}-$ supp- 42

\section{Keywords:} miRNA miR-16 Flow Cytometry Breast Cancer miR-34a

Apoptosis

\begin{abstract}
Introduction It has been shown that miR-34 family (miR-34a/b/c) are direct targets of p53 and miR-34a is expressed at a higher level than the other members. MiR-34 family are involved in induction of apoptosis and cell cycle arrest. The anti-apoptotic BCL2 gene is also a miR-16 target. It has been shown that miR-16 is either deleted or downregulated in cancer and therefore miR-16 has been suggested as a possible 'hot spot' in cancer transformation. Based on the functional similarities between miR-34a and miR-16 we aimed to investigate whether overexpression of miR-34a and miR-16 can cooperate in induction of cell cycle arrest and apoptosis in human breast cancer cells.

Materials and Methods: MDA-MB-231 and SK-BR-3 human breast cancer cell lines were cultured and transfected with synthetic hsa-miR-16 mimic, hsa-miR-34a mimic, or both using HiPerFect transfection reagent. A mock transfection was also included as the control. The cells were re-transfected after 72 hours and harvested at the 7 th day. Analysis of apoptotic cell population was performed using Annexin V-FITC/PI staining and flow cytometry. The data were analyzed by FlowJo software.

Results: In both cell lines, either miR-16 or miR-34a induced apoptosis individually. Meanwhile, transfection of the cells with both miR-16 and miR-34a showed a significant increase in apoptotic rate which was higher than individual apoptotic rates of either miR$34 \mathrm{a}$ or miR-16. Therefore, miR-34a and miR-16 synergistically induced apoptosis in human breast cancer cells.

Conclusions: Our results are indicating that miR-34a and miR-16 can cooperate in induction of apoptosis in breast cancer cells. The synergistic effect of these miRNAs on the apoptotic cancer cell population may have potential applications in inhibition and treatment of invasive cancer types.
\end{abstract}

